\title{
What is the relationship between parental support, self-efficacy and physical activity in young students? A pilot cross-sectional study
}

\author{
Alberto Ruiz-Ariza ${ }^{1 \mathrm{a}}$, Manuel de la Torre-Cruz ${ }^{1}$, Sebastián López-Serrano ${ }^{1}$, and Emilio J. \\ Martínez-López ${ }^{1}$ \\ ${ }^{1}$ Faculty of Humanities and Education Sciences, University of Jaen, Campus Las Lagunillas s/n, Jaen, \\ Spain
}

\begin{abstract}
This study examined the relationships between parental support for physical activity, weekly Physical Activity (PA) practice frequency, and self-efficacy expectations to overcome barriers to participation. A total of 335 adolescents took part in this pilot cross-sectional study. The results show that boys and girls who attribute parents to a high level of instrumental support, modeling and behavioral limitation are allocated to higher number of days per week to perform the physical-sport activity during a minimum interval of sixty minutes and were more self-efficacious in their practice. Likewise, the frequency of practice of the activity and self-efficacy were higher in the case of boys.
\end{abstract}

Keywords: Physical activity, parental support, self-efficacy.

\section{Introduction}

The practice of physical activity (PA) maintains a positive relation with the physical and psychological health of children and adolescents [1]. Despite of these benefits, the percentage of young people who perform the recommended account of 60 daily minutes of physical-sport activity at moderate to vigorous intensity, has decreased in the last two decades $[2,3]$.

In this regard, Bandura's [4] cognitive-social theory, suggests that the provision of social support increases the self-efficacy to overcome obstacles and the possibility to be physically active. One of the main sources of support is in the family [5]. The different kinds of parental support (logistic, modeling, establishment of limits) could differentially affect the practice of PA, and the examination of their influence could further clarify these relationships $[6,7]$.

Thus, the objective of this study was to examine the relationships between parental support, weekly frequency of PA and expectations of self-efficacy.

\footnotetext{
${ }^{\text {a }}$ Corresponding author: arariza@ujaen.es
} 


\section{Method}

A total of 335 students ( girls $=175,52.3 \%)$ with ages between 12 and 17 years $(\mathrm{M}=13.8$; $\mathrm{SD}=1.36$ ) participated in this pilot cross-sectional study.

\subsection{Process}

The parents, directors and teachers were informed about the purpose of this study. Informed consent was obtained from parents. The different questionnaires were applied during Physical Education classes. Participants had 50 minutes to perform the tests. The procedure for obtaining data took into account the current Spanish legislation for research in humans (Royal Decree 561/1993 on clinical trials), the law on protection of personal data (Organic Law 15/1999) and the Declaration of Helsinki.

\subsection{Instruments}

To assess parental support, a Spanish version of the "Activity Support Scale, ACTS" proposed, by Davison [8], was used. This scale is composed of nine items grouped into three factors: instrumental support ("It took me to places where I could perform PA"), modeling ("It encouraged me to be physically active / acting as an example and being a model") and establishing limits to sedentary behavior ("I have limited the daily amount of hours playing video games"). The participants had to indicate along a Likert four-point scale the degree of agreement $(1=$ total disagreement, $4=$ total agreement $)$. The reliability indexes obtained for this study sample were .73 for the instrumental support dimension, .71 for the modeling dimension, and .79 for the limit dimension to sedentary behavior.

The MVPA "Moderate-to-Vigorous PA" scale [9] reports the number of days during the previous week and any one week in which participants performed moderate to vigorous intensity PA for at least 60 minutes. A value of five or higher corresponds to the inclusion of the young person in the physically active category. The reliability index of the two items in the study sample was .84.

Self-efficacy for the practice of PA was evaluated using an eight-item questionnaire developed by Dishman et al. [10]. Some examples of the items included were: "I am able to engage in PA in my free time regardless of how busy or busy the day" and "I am able to ask my parents or other adults to engage in PA with me during my free time". The items were valued along a Likert scale in which the participant had to indicate their degree of agreement with the different affirmations. The range of values ranged from 1 (totally disagree) to 5 (totally agree). A single factor was obtained that explained $49 \%$ of the variance of the scores obtained. The reliability index for this study sample was .84 .

\section{Results}

\subsection{Relationship between parental support and frequency of PA}

Three univariate analyzes of covariance (ANCOVAs) were performed to know the relationship between perceived family support for the practice of physical-sport activity and the reported frequency of weekly practice of MVPA. Because previous research reveals that body mass index and chronological age have a negative relationship with the enjoyment and frequency of PA practice, these two variables were included as covariates. 
The first of the analyses revealed a statistically significant main effect of instrumental parental support, $\mathrm{F}_{(1,38)}=51.18, \mathrm{p}<.001, \eta 2=.13$ and the participant's gender, $\mathrm{F}_{(1,389)}=$ $26.79, \mathrm{P}<.001, \eta 2=.07$ and the weekly frequency of PA.

On the other hand, the participants who attributed a greater practice of physical-sport activity to their parents showed a higher frequency of weekly practical activity $(M=3.59$ vs $M=2.79)$. Likewise, boys were more frequently involved than girls in this type of activity $(\mathrm{M}=3.58$ vs $\mathrm{M}=2.83)$.

The third of the analyses revealed, as in the previous cases, that the frequency of practice of PA was higher in young people who attributed to their parents a greater restriction to sedentary behavior $(\mathrm{M}=3.41$ vs $\mathrm{M}=2.97)$.

\subsection{Relationship between parental support and perceived self-efficacy for the practice of PA}

The analyses revealed that the highest perceived self-efficacy scores for PA were observed in those participants who attributed to their parents a high degree of instrumental support $(\mathrm{M}=29.38$ vs. $\mathrm{M}=24.01)$.

The second analysis showed the existence of a statistically significant main effect of the modeled variable, $\mathrm{F}_{(1,371)}=46.25, \mathrm{p}<.001, \eta 2=.1$. Adolescents who considered that both mothers and fathers were frequent models of PA to imitate reported higher self-efficacy expectations for PA $(\mathrm{M}=29.15$ vs $\mathrm{M}=24.03)$.

Finally, the last of the analyses showed a statistically significant main effect of the parental limitation of the sedentary behaviors, $F_{(1,412)}=13.48, p<.001, \eta 2=.04$. The selfefficacy perceived for the practice of PA was greater in the group of adolescents who perceived in their parents a greater imposition of limits on the performance of sedentary behaviors $(\mathrm{M}=27.56$ vs $\mathrm{M}=24.45)$.

\section{Discussion}

The results show that boys and girls who attribute parents to a high level of instrumental support, modeling and behavioral limitation are allocated to higher number of days per week to perform the physical-sport activity during a minimum interval of sixty minutes and were more self-efficacious in their practice. These results are in line with those reported by previous research [6, 11-12]. Likewise, the participants who considered their parents a greater modeling of behaviors associated to the practice of PA expressed to be more active and more self-efficacious in relation to their equals whose attributed parental modeling was smaller. In consonance with this finding, several authors examined the relationship between parents and children weekly PA by accelerometers [13]. The results revealed that modeling showed a positive relationship, although weak, between the practice of PA performed by parents and children. However, the relationship was higher when parents were divided between those who met the international recommendations of daily PA practice (at least 30 minutes at moderate to vigorous intensity) and those who did not this PA amount.

\section{Conclusions}

It is possible that the socialization processes analyzed in this study have a bi-directional nature where the parental practices associated with PA affect the attitudes and behaviors of the children while the latter offer certain keys to their parents who regulate the types and levels of family actions aimed at promoting participation in activities of a physical and sports nature. On the other hand, the use of self-report measures is an additional limitation 
to this study. Evaluating possible associations by including objective measures of PA (for example, using the use of accelerometers) could become an interesting route of new researches.

\section{References}

1. F.K.W. Ho, L.H.T. Louie, C.B. Chow, W.H.S. Wong, P. Ip, BMC Pediatrics, 15, 48 (2015)

2. K.K. Davison, G.D. Deane, Social Science Medicine, 70, 518 (2010)

3. D.J. Graham, K.W. Bauer, S. Friend, D.J. Barr-Anderson, D. Neumark-Sztainer, Journal of Physical Activity and Health, 11, 51 (2014)

4. A. Bandura, Self-efficacy: The exercise of control. (New York, Freeman, 1997)

5. Y. Laird, A. Fawkner, P. Kelly, L. McNamee, A. Niven, International Journal of Behavioral Nutrition and Physical Activity, 13, 79 (2016)

6. S.J. Sebire, A.M. Haase, A.A. Montgomery, J. McNeill, R. Jago, Journal of Physical Activity and Health, 11, 734 (2014)

7. K.K. Davison, R. Jago, Medicine \& Science in Sports and Exercise, 41, 1816 (2009)

8. K.K. Davison, Journal of Physical Activity and Health, 1, 363 (2004)

9. J.J. Prochaska, J.F. Sallis, B. Long, Archives of Pediatrics and Adolescents Medicine, 155, 554 (2001)

10. R.K. Dishman, D.P. Hales, J.F. Sallis, R. Saunders, A.L. Dunn, A.L. Bedimo-Rung, K.B. Ring, Journal of Pediatric Psychology, 35, 72 (2010)

11. S.G. Trost, P.D. Loprinzi, American Journal of Lifestyle Medicine, 5, 171 (2011)

12. M. Verloigne, J. Veitch, A. Carver, J. Salmon, G. Cardon, I. Bourdeaidhuij, A. Timperio, BMC Public Health, 14, 996 (2014)

13. J. Jago, J.L. Thompson, S.J. Sebire, L. Wood, L. Pool, J. Zahra, D.A. Lawlor, International Journal of Behavioral Nutrition and Physical Activity, 11, 54 (2014) 